\title{
Research of Mobile Robot Path Planning Based on Genetic Algorithm
}

\author{
Jian ZHANG \\ (School of Computer Engineering, Anhui Sanlian University, Hefei 230601, Anhui,China) \\ 626742382@qq.com
}

Key words: Mobile robot; Genetic algorithm; Cyclotron curve Smooth; Path planning

\begin{abstract}
Path planning is an important task in the field of robot. A new method of dividing genetic algorithm(GA) process is proposed,which is different from the traditional method that considers smoothness in GA process. After calculating a path using simplified GA method with a variable length coding, a new type of clothoid curve with wipe parameter is introduced to smooth the sharp turns. In the entire path planning process, the parameter can be chosen adaptively to generate a robot's walk path as soon as the barrier's coordinate is entered. The simulation results show that the method of separating smooth process from GA process simplifies GA method.The proposed method could efficiently decrease the path length as well as increase the path smoothness.
\end{abstract}

\section{Introduction}

\section{Background and research status}

Robot path planning is an important topic in the field of robotics, it is actually a complex nonlinear programming problem, which mainly solves in the presence of obstacles in the environment for the robot to find out a collision - free path to walk from the beginning to the end, the path should meet the length is short, time is short, the smoothness of higher standard. Robot path planning method plays a vital role in the safety and efficiency of the robot.

The current robot path planning method for a graph search method, with the evaluation function of the Dijkstra algorithm ${ }^{[1]}$, fuzzy reasoning method, the artificial potential field method, artificial neural network method, random bitmap method etc., these algorithms have their respective advantages, but overall still exist problems such as low adaptability and complex algorithm.

As a kind of optimization algorithm, genetic algorithm ${ }^{[2]}$ has been widely used in the robot path planning, which shows good robustness in the complex nonlinear optimization problems. The algorithm defines the genetic operation such as smoothing and truncated to smooth the path of large angle, these operations can locally adjust the robot to walk the path angle, taking account of the effect of the path of the smoothness problem, but in different degree increased the complexity of the genetic algorithm. Is proposed in this paper introducing cyclotron spirals that separates the genetic process and smoothing method, to improve the path smooth, shorten the path length of the requirements, but also reduces the genetic algorithm complexity. 


\section{Swirling spiral of knowledge presentation \\ Swirling spiral}

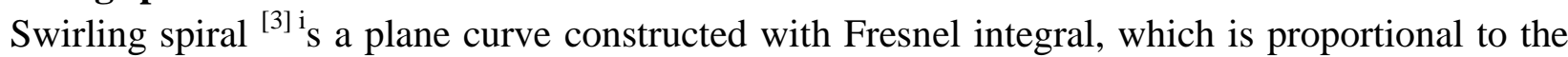
arc length and curvature. Here introduces a band shape parameter $\lambda(0 \leq \lambda \leq 1)$ promotion of the Fresnel integral, with this integral structure swirling spirals, when $\lambda=0.5$ when it becomes a standard spiral maneuver.

Definition of the curve

$$
\mathrm{r}(\theta ; \lambda)=(\mathrm{x}(\theta), \mathrm{y}(\theta))=\alpha(\mathrm{C}(\theta ; \lambda), \mathrm{S}(\theta ; \lambda))
$$

Becoming the promotion of swirling spiral (the cyclotron spiral). Wherein the scaling factor $\alpha$ is a positive number, shape factor $0 \leq \lambda<1, \theta$ represents Cutaway deteriorated, $C(\theta ; \lambda)$ and $S(\theta ; \lambda)$ with shape parameter $\lambda$ is the promotion of the Fresnel integral.

$$
\mathrm{C}(\theta ; \lambda)=\frac{1}{\sqrt{2 \pi}} \int_{0}^{\theta} \frac{\cos \mu}{\mu^{\lambda}} d \mu, \mathrm{S}(\theta ; \lambda)=\frac{1}{\sqrt{2 \pi}} \int_{0}^{\theta \sin \mu} \frac{\mu^{\lambda}}{\mu^{\lambda}}
$$

\section{Swirling spiral even ${ }^{[3,4]}$}

Definition of dual swirling spiral (clothoid pair) is composed of two spiral maneuver

$$
\mathrm{r}(\theta ; \lambda)=\mathrm{P}_{\mathrm{i}}+\alpha_{\mathrm{i}} \mathrm{C}(\theta ; \lambda) \mathrm{T}_{\mathrm{i}}+\alpha_{\mathrm{i}} \mathrm{S}(\theta ; \lambda) \mathrm{N}_{\mathrm{i}}, 0 \leq \theta \leq \theta_{i}, \mathrm{i}=0,1
$$

Combination, where $P_{i}, i=0,1$ are their starting point, $T_{i}$ is a unit tangent vector at the beginning, $\mathrm{Ni}$ is the unit normal vector at the start. This requires maintaining $\mathrm{G}^{2}$ between two consecutive spiral swirling, i.e. they coincide with the end point, and parallel to the tangent vector at the end point of curvature equal to the unsigned.

The new robot path planning algorithm and genetic algorithm-based cyclotron spirals

Traditional genetic algorithm on the robot path to improve the smoothness of the problem, take most of the fitness function rewritten or increased genetic manipulation methods. In order to reduce the complexity of the genetic algorithm, flexible adjustment path in the corner, we put forward a smoothing process and the process of genetic algorithm to separate approach, each of these two will be described in detail.

\section{Genetic Algorithm Description}

The robot simplified as particle, assuming that the obstacle is a convex polygon. The outward expansion of the boundaries of each obstacle a safe distance, the formation of new obstacles, so that even if a new obstacle vertex path coincides with the original obstacle actually maintain a safe distance.

\section{Encoding}

Text taken float variable length encoding, the chromosome sequence Cartesian path node configuration

Population initialization, In order to increase the diversity of the population, the paper taken randomly generated initial

\section{population}

fitness function

Has been an obstacle in front of the border to expand outward representation of the environment a safe distance, and later will introduce dual swirling spiral path smoothing operation, it only needs to include the fitness function and determine whether the length of the path and obstacles It was touching.

Fitness function is: 
$\mathrm{F}=\mathrm{N}-\left(\alpha_{1} \sum_{i=1}^{n} d_{i}+\alpha_{2} \times m\right)$

Wherein ,di represents the length of the path of subparagraph $\mathrm{i}, \sum_{i=1}^{n} d_{i}$ the total path length. $\mathrm{M}$ is the number of paths through the obstacle. $\alpha_{1} 、 \alpha_{2}$ Proportionality constant. $N$ is a large integer, the higher the fitness of individuals better performance.

\section{Genetic Manipulation}

(1) Selection Operator: This article is taken roulette selection method implementation operation.

(2) Crossover: This article take a single point crossover method. Since the variable-length coding, so the two chromosomes can not cross the same position. If the individual length of the new production exceeds the maximum length of chromosomes, then discarded.

(3) Mutation Operator: individuals with a certain probability variation range for the entire space, in order to increase the diversity of the population.

\section{genetic algorithm termination conditions}

When the population evolution generation reaches a predetermined upper limit, terminate the genetic process.

\section{based smoothing operation swirling spirals}

It said genetic algorithm can generate high efficiency collision-free path to a polyline optimum, and now with the introduction of a class of shape parameters promotion swirling spiral, polyline paths have been generated by the smoothing operation, and adaptively selecting parameters yielding the final smooth path. First introduced following the introduction of a smoothing operation swirling spirals in a single node, and then describes the smooth operation of the plurality of nodes. This chapter to use the knowledge about the swirling spirals have been given in the introduction.

To simplify the algorithm, in order to obtain an optimum high efficiency collision-free path polyline, genetic processes previously described consider temporarily eliminating the smoothness of the path, then the path may contain a greater angle, such as Figure 2.1 the angle $\alpha$ and angle $\beta$. Preset according to the specific circumstances of a security robot corner corner in order to determine the size of each node on this path, when it exceeds a predetermined safety angle, this article will introduce the above-mentioned promotion swirling spiral even its smooth operation ${ }^{[5]}$.

$\mathrm{P}_{\mathrm{i}}$ may wish to set point angle $\alpha$ exceeds the safety point of view, the point needs to be a smooth operation. Figure 2.2, the connection $\mathrm{P}_{\mathrm{i}}$ and two adjacent nodes selected points $\mathrm{A}$ and $\mathrm{B}$, and $\mathrm{A}, \mathrm{P}_{\mathrm{i}}$, $\mathrm{B}$ three points seen as control points, select the shape parameter $\lambda$, generating a swirling spiral even as new path instead of the original polyline path. Thus, with the purpose to achieve a smooth corner, but can also shorten the path length.

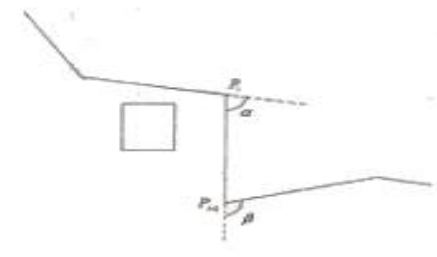

Figure 2.1 Large angle in a broken line 


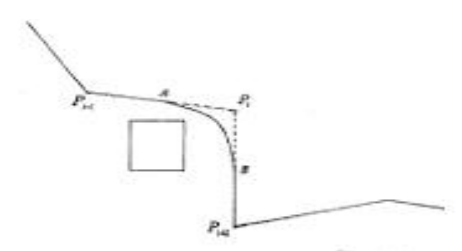

Figure 2.2 Smooth operation at large angle

If the resulting swirling spiral obstacle collision even need to be adjusted. Since $\lambda=0.5$ by the restrictions by moving control points to avoid obstacles, without having to move any of the control point, simply increase the value of the shape parameter $\lambda$ can make swirling spiral even closer control polygon, thus avoiding its control open obstructions. Note that, when the larger shape parameter $\lambda$, whirling spirals even more close to the original polyline path, turn against security robots. From this point of view, the shape parameter $\lambda$ should be as small as possible, and the path length at this time will be shortened. However, when $\lambda$ is too small, swirling spirals even away from the control polygon, it is likely to impact the obstacle. Therefore, this paper is in operation, so that $\lambda$ $(0 \leq \lambda \leq 1)$ value from the initial value of 0.1 started to increase in steps of 0.1 adaptively select the shape parameter $\lambda$ value, making it a condition to ensure the Theorem and the resulting path no obstacle collision minimum ${ }^{[6]}$.

When the path contains a plurality of large angle, turn smoothing appeal for each corner. Shown in Figure 2.3, you may assume $\mathrm{P}_{\mathrm{i}}$ and $\mathrm{P}_{\mathrm{i}+1}$ Chu corner exceeded the safety point of view, respectively, they were smoothing operation. Smoothing Pi point same as above, the following of $P_{i+1}$ point smoothing operation. Select the midpoint $C$ in $P_{i+1}$ and $P_{i+2}$ connection to $B, P_{i+1}$, In $B, P_{i+1}, C$ line path control point generating spiral cyclotron point pairs instead of the original $\mathrm{P}_{\mathrm{i}+1}$.

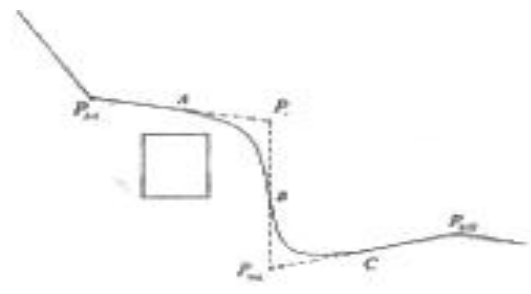

Figure 2.3 Smooth handling of multiple rotations

As the transition curve and circular arc, and a straight line and a straight line or arc between the swirling spirals and even control polygon combination generated spline curve to achieve $\mathrm{G}^{2}$ continuous. So even spiral maneuver can be well embedded into the dogleg path GA obtained, so that the path of the new generation can maintain a high continuity. Meanwhile, the smooth operation of each corner independent of each other, that they can be independent of the shape parameter values, so you can adjust the shape parameters to the local shape of the path, flexible and convenient. This smoothing operation can improve the security of the robot when cornering, but also shorten the length of original polyline path ${ }^{[7]}$.

\section{Simulation results}

Path planning method designed in this paper, simply enter the coordinates of the environment, it can adaptively obtain the final smooth path. In order to verify the effectiveness of the algorithm, the following were special obstacles, obstacles and irregular rules more obstacle environment were three groups of simulation experiments.

As shown in special obstacles 3.1 (a), in which three elongated object is an obstacle, the starting point of the robot is $(0,50)$, end point $(100,50)$, although this environment obstacles rare, 
distribution of obstacles large and unique. Figure 3.1 (b) of the rules of multi-obstacle environment, the figure is small squares arranged in an orderly one obstacle, the robot starting point for the $(0,30)$, end point $(100,80)$, this environment obstacles distribution, but in larger quantities. Figure 3.1 (c) an irregular multi-obstacle environment, the figure scattered polygons one of the obstacles, the robot starting point for the $(0,0)$ and end point $(100,100)$, the shape of the obstacle in this environment irregular distribution of clutter and in larger quantities.

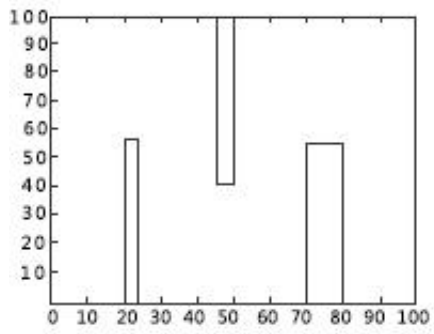

Figure 3.1 (a) special multi-obstacle environment

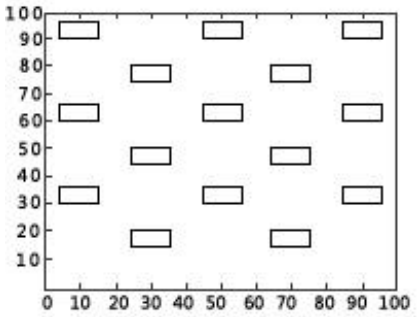

Figure 3.1 (b) rules for multi-obstacle environment

Figure 3.1 Three different experimental environment

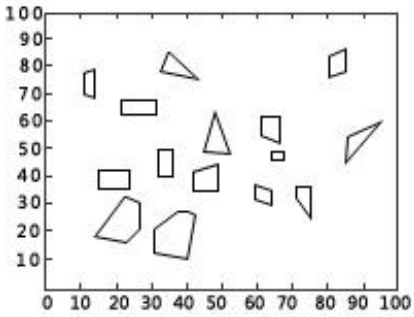

Figure 3.1 (c) irregular multi-obstacle environment

First, the simulation experiments in a special obstacle environment, shown in Figure 3.2. Due to the special nature of the obstacle, the traditional path of the resulting path planning approach based solely on genetic algorithms will inevitably have a greater angle. As shown in Figure 3.2 (a) of this article is a line diagram of the genetic algorithm. The resulting robot obstacle avoidance walking path, including node $\mathrm{P}_{1}, \mathrm{P}_{2}, \mathrm{P}_{3}, \mathrm{P}_{4}, \mathrm{P}_{5}$, wherein $\mathrm{P}_{2}, \mathrm{P}_{3}$ and $\mathrm{P}_{4}$ are corner exceeds a predetermined safety angle of 90 degrees, they should be a smooth operation.

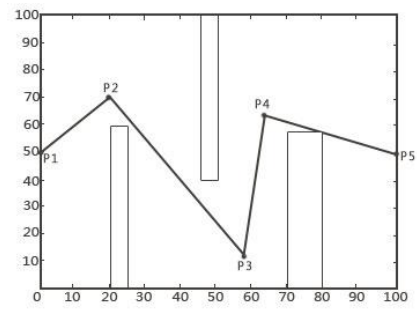

Figure 3.2 (a) of genetic algorithm dogleg path

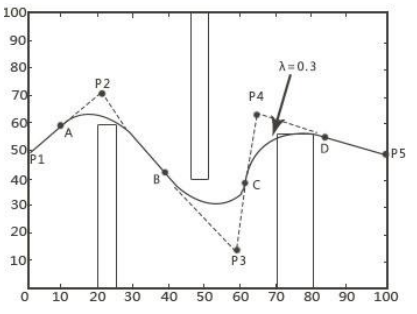

Figure 3.2 (b) adaptive selection of process parameters

Figure 3.2 Special obstacle environments experiment

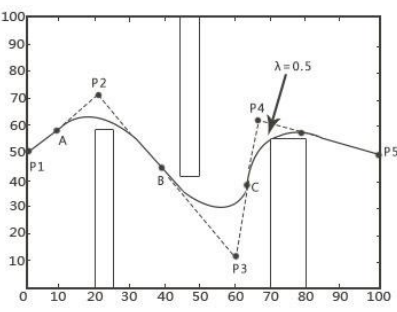

Figure 3.2 (c) of the final smooth path

FIG3.2 (b), the midpoint taken $\mathrm{P}_{1} \mathrm{P}_{2} \mathrm{~A}$, taken $\mathrm{P}_{2}, \mathrm{P}_{3}$ midpoint $\mathrm{B}$, taken $\mathrm{P}_{3}, \mathrm{P}_{4}$ of the midpoint of the $\mathrm{C}$, take $\mathrm{P}_{4}, \mathrm{P}_{5}$ of the midpoint of the $\mathrm{D}$, with $\mathrm{A}, \mathrm{P}_{2}, \mathrm{~B}$ is control point $\mathrm{P}_{2}$ to $\mathrm{B}, \mathrm{P}_{3}, \mathrm{C}$ is the control point $\mathrm{P}_{3}$ to $\mathrm{C}, \mathrm{P}_{4}, \mathrm{D}$ at the control point $\mathrm{P}_{4}$. Respectively points adaptively selected shape parameter, $\lambda(0 \leq \lambda \leq 1)$ beginning from 0.1 to 0.1 steps increase. After calculation, when the shape parameter three nodes were taken $\lambda=0.7, \lambda=0.5, \lambda=0.3$, the theorems were first meet a minimum swirling spiral shape parameter even producing conditions. Figure 3.2 (b) of the curve is first generated swirling spiral even at each node, the dotted line for the control polygon, polyline that is the original path.

Calculated that, at this point $\mathrm{P}_{2}$ and $\mathrm{P}_{3}$ at first generated swirling spiral even have been able to avoid obstacles, it does not increase the value of its shape parameters. The $\mathrm{P}_{4}$ at the point when $\lambda=0.3$ at $\lambda$ values even though meeting the swing spiral formation conditions, but the path will be generated by a collision barrier, need to continue to increase. Calculated until $\lambda=0.5$, the resulting swirling spiral even without collision obstacle, as shown in 3.2 (c) as shown, so the final $\mathrm{P}_{4}$ is taking shape parameter $\lambda=0.5$.

A second set of experiments run in a multi-path planning barriers to environmental regulations in. As shown in Figure 3.3, is entered after more obstacle environment and ultimately obtained a smooth path adaptation. Figure polyline as a path partially genetic algorithms, including nodes $\mathrm{p}_{1}$, $\mathrm{P}_{2}, \mathrm{P}_{3}$. Curve is generated on the point $\mathrm{p}_{2}$ after whirling spiral smoothing operation, the control 
point $\mathrm{P}_{2}, \mathrm{P}_{1} \mathrm{P}_{2}$ midpoint $\mathrm{A}$ and $\mathrm{P}_{2} \mathrm{P}_{3}$ midpoint $\mathrm{B}$, adaptive selection shape parameter of the result is 0.4 .

The third group of irregular path planning experiments in multi-obstacle environment to run, Figure polyline as a path partially genetic algorithms, including nodes $\mathrm{P}_{1}, \mathrm{P}_{2}, \mathrm{P}_{3}$. Curve is generated on the point $\mathrm{P}_{2}$ after whirling spiral smoothing operation, the control point $\mathrm{P}_{2}, \mathrm{P}_{1} \mathrm{P}_{2}$ midpoint $\mathrm{A}$ and $\mathrm{P}_{2} \mathrm{P}_{3}$ midpoint $\mathrm{B}$, adaptive selection shape parameter of the result is 0.6.

Will be apparent from the above simulation results, in three different environment obstacles, whirling spiral even polyline paths smoothing operation after they have reached the effect of smoothing the path, the path of total length has also been a certain degree of shortening. Especially in the case of the first group of special obstacle distributed simulation results, to overcome the problem of large angular traditional genetic algorithm difficult to avoid. The smooth operation of the genetic algorithm to separate the practice can also reduce the complexity of the genetic algorithm itself.

\section{Summary}

Traditional genetic algorithm on the robot path to improve the smoothness of the problem, take most of the fitness function rewritten or increased genetic manipulation method, this article is the genetic algorithm process and path smoothing process using separate ways. After the genetic algorithm to generate optimum path, the introduction of a new class of swirling spiral shape parameters even with its smooth operation. Simply enter the entire path of the planning process can be an obstacle coordinates adaptive selection parameters to generate a smooth path, and just change the shape parameters can be flexibly adjusted path obstacle coordinate distance. Simulation results show that the introduction of swirling spirals will separate the genetic process and smooth operation of the method can to improve the smoothness of the path, shorten the path length requirements, but also reduces the complexity of the genetic algorithm itself.

\section{Acknowledgements}

2016 annual university service robots Collaborative Innovation Center Fund Project: Application (xtcx2016002) ZigBee technology in the intelligent robot systems. 2013 Provincial Department of Education University Natural research: Based on the ZigBee wireless network technology (KJ2013B090) in the intelligent traffic signal control.

\section{references}

[1] Sun Guang, Li Chungui, Wang Qin. robot path planning based on improved genetic algorithm [J] Automation and Instrumentation, 2009, (6): 5-7.

[2] Liang Xian LI. autonomous robot soccer path planning and obstacle avoidance strategy research

[D] Jiaozuo: Henan University of Technology, 2011.

[3] Hao Bo, Qin Lijuan, Jiang Ming Yang. Mobile Robot Path Planning Method Based on Improved Genetic Algorithm [J]. Computer Engineering and Science, 2010, (7): 104-107.

[4] Dantie Feng. Computer Simulation Improved Genetic Algorithm in mobile robot path planning [J], 2011, (4):.. 193-195.

[5] Liao Weiqiang, Li Zhenyu.soccer robot path based on genetic potential field of planning [J] Jimei University: Natural Science Edition, 2009, (2): 179-184.

[6] Dingchao Pu.given over study [D] Path Planning for Mobile Robot Based on Genetic Algorithms Hefei: Hefei University of Technology, 2010. 
[7] Gang Li, DaoTong Guo, Zou Yang. robot path planning based on improved genetic algorithm and simulation [J] Computing Technology and Automation, 2015, (2): 24-27. 